\title{
Teleconsultation experience with the idiopathic inflammatory myopathies: a prospective observational cohort study during the COVID-19 pandemic
}

\author{
R. Naveen ${ }^{1}$ (D) T. G. Sundaram ${ }^{1}$ (D) Vikas Agarwal $^{1}$ (I) $\cdot$ Latika Gupta $^{1}$ (D)
}

Received: 23 September 2020 / Accepted: 21 October 2020 / Published online: 4 November 2020

(c) Springer-Verlag GmbH Germany, part of Springer Nature 2020

\begin{abstract}
Teleconsultation has assumed a central role in the management of chronic and disabling rheumatic diseases, such as the idiopathic inflammatory myopathies (IIM), during COVID-19. However, the feasibility, challenges encountered, and outcomes remain largely unexplored. Here, we describe our teleconsultation experience in a prospectively followed cohort of adult and juvenile IIM. 250 IIM enrolled into the MyoCite cohort (2017-ongoing) were offered the option of audio/visual teleconsultation using WhatsApp during the nationwide lockdown. Clinical outcomes (major/minor relapse) and prescription changes were compared between IIM subsets. Socio-demographic and clinico-serological characteristics of those who sought teleconsultation were compared with those who did not. 151 teleconsultations were sought over a 93 day period by $71(52.2 \%)$ of 136 IIM (median age 38 years, F:M 4.5:1). Nearly one-third (38\%) consulted on an emergency basis, with voice consultations being the primary medium of communication. Over a quarter (26.8\%) reported relapse (15.5\% minor, $11.3 \%$ major), these being more common in JDM [71.4\%, OR 8.9 (1.5-51)] as compared with adult IIM, but similar across various antibody-based IIM subtypes. Patients who relapsed required more consultations [2(2-3) vs 1(1-2), $p 0.009]$. The demographic and socioeconomic profile of the patients seeking consultation $(n=71)$ was not different from those who did not $(n=65)$. Voice-based teleconsultations may be useful to diagnose and manage relapses in IIM during the pandemic. Patient education for meticulous and timely reporting may be improve care, and larger multicentre studies may identify subsets of IIM that require greater care and early tele-triage for effective management of the condition.
\end{abstract}

Keywords Teleconsultation $\cdot$ COVID-19 $\cdot$ Triage $\cdot$ Remote consultation $\cdot$ Myositis

\section{Introduction}

The coronavirus disease 2019 (COVID-19) pandemic has disrupted conventional practice of medicine and called for a revised model of socially distanced health care. Virtual consulting assumes a larger importance for management of chronic and disabling rheumatic diseases (RDs), such as the idiopathic inflammatory myopathies (IIM). With a longprojected duration of the global pandemic, Teleconsultations have become the preferred means of patient-doctor communication and monitoring. In a recent global survey among

Latika Gupta

drlatikagupta@gmail.com

1 Department of Clinical Immunology and Rheumatology, Sanjay Gandhi Postgraduate Institute of Medical Sciences, Lucknow, India patients with IIM, the use of audio-visual communication for a teleconsultation was supported by most (70\%), while telephonic consultations were also acceptable to a majority (52\%). [1] While teleconsultations are fraught with logistic challenges and penetrance issues in low middle- income countries (LMIC), the safety, cost benefit and convenience may account for greater preference (82\%) for remote consultation by rheumatology outpatients, a large proportion of whom may be scheduled for routine assessment of stable disease, as reported in a recent Polish study. In fact, this model may be sustainable post-pandemic, given that merely $8.2 \%$ wished to discontinue the tele-services post pandemic [2].

However, the situation in chronic RDs, such as IIM, may be met with additional challenges due to more frequently hampered mobility, distinctive need to measure muscle strength objectively, varied organ system involvement, and frequent need for admissions. [3] In a LMIC, the availability of smartphones and internet access in rural areas may 
be limited. Language diversity and poor literacy is another impediment to successful communication, and a major concern while delivering care remotely. We recently observed that IIM is under-represented among teleconsultations for RDs, but over-represented among admissions. [3, 4] Rheumatologists in various countries have adapted their patient services according to the pandemic situations, with specific focus being minimizing patient health care personnel contact, ensuring safe practices, maximising teleconsultation services, and admitting only when utmost needed [5-7].

Remote consultation does well for chronic diseases where patient-reported-outcome measures for measuring disease activity, damage and functional loss are validated and obtainable. To the same effect, teleconsultation has been extensively studied in rheumatoid arthritis, systemic sclerosis, fibromyalgia, osteoarthritis and juvenile idiopathic arthritis. $[4,8]$ However, the feasibility, challenges encountered, and patient-reported outcomes in IIM remain largely unexplored. Here, we describe our teleconsultation experience in a prospectively followed cohort of adult and juvenile IIM.

\section{Methods}

\section{Patient population}

Of 550 registered patients with IIM (1989-2020), 250 are enrolled into a cohort study (December 2017 to present) at a rheumatology tertiary-care center. [9] These patients were were contacted by email (sent on 24th March 2020) and the social messaging application, WhatsApp ${ }^{\mathrm{TM}}$ after the implementation of a nationwide lockdown in India on 23 March 2020. In view of travel restrictions, patients were intimated to avail teleconsultation facilities through a previously designated helpline number, on their previously allotted appointment dates. Patient could contact the rheumatology team over a phone-call, WhatsApp text, instant message or email. All queries were responded twice a week, on previously designated clinic days (wednesdays and fridays). 136 of the 250 received the message, while the rest did not (the reason could be due to change of phone numbers, termination of follow-up, or death of the patient) (Fig. 1a). We used the STROBE checklist for reporting methods and results [10].
$\mathbf{A}$

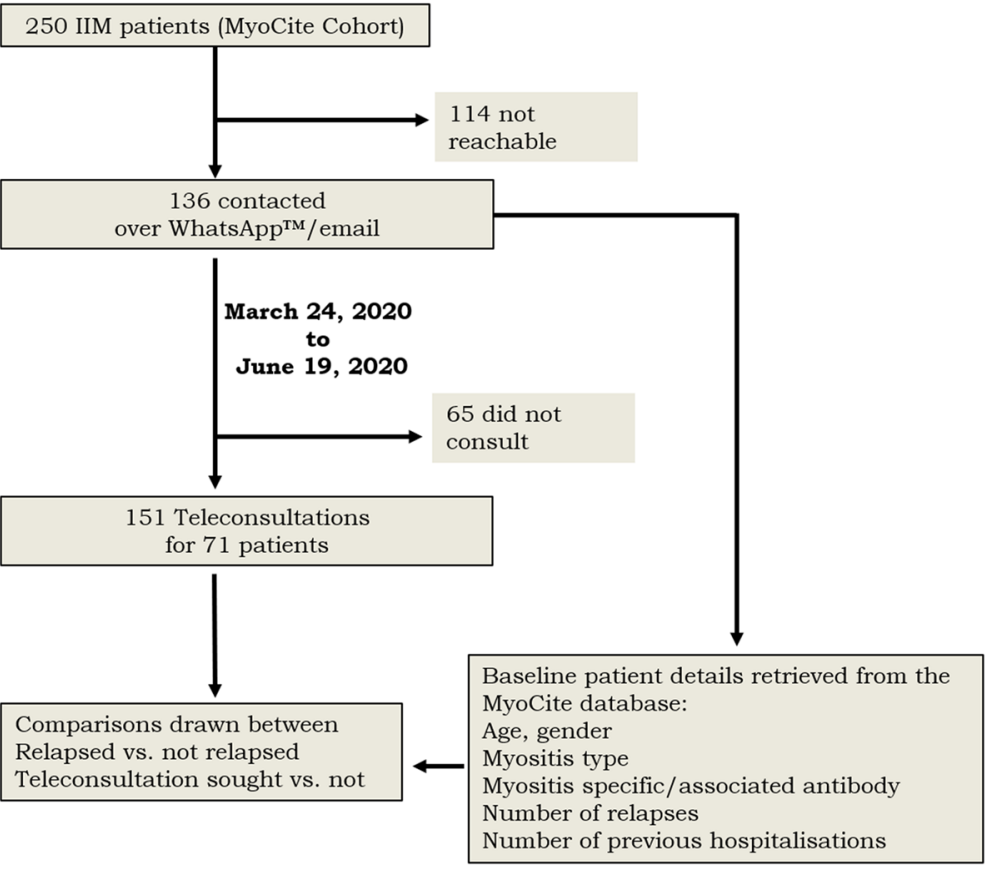

\section{B}

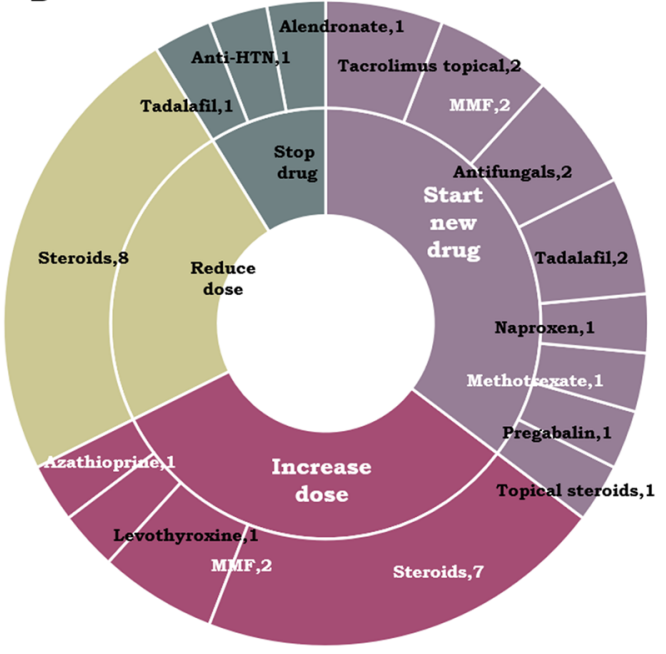

$\mathbf{C}$

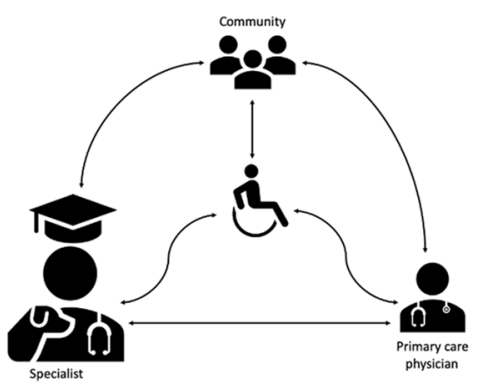

Fig. 1 a Methods flow chart. b Drug interventions. c Proposed three tier health structure 


\section{Data collection}

When dealing with new skin lesions, imaging or laboratory results, the patients were requested to share photographs or documents on WhatsApp. When the issues could not be sorted out through audio calling or messages, the patients were could opt for video-calling on WhatsApp. Data were collected from 24 March 2020 till 19 June 2020.

The number of consults per patient, time since the nationwide lockdown to the first call, and time between consults was calculated. Prescriptions and ancillary advice were tabulated. Challenges encountered and proposed solutions were also recorded. Patient demographics (age, sex, location by pin-code) and socio-economic strata (annual income of the family) were retrieved from database archive $[9,11,12]$. Details on baseline characteristics, such as myositis subtype, and myositis-specific and -associated antibodies (Line immunoassay, Lubeck, Germany), were also obtained from clinical and biorepository archives. Definitions for organ involvement and type of IIM are as previously described [9, 12].

Relapse was defined as per physician judgement based on two or more among the following: worsening muscle weakness or myalgias, new rashes thought to be consistent with relapse of IIM, increased muscle enzymes, worsened physical function deemed to be related to muscle disease and an improvement with increased dose of glucocorticoids. A major relapse was defined as those managed with glucocorticoid dose equivalent of $0.5 \mathrm{mg} / \mathrm{kg}$ body weight or more, while those requiring a lower dose of glucocorticoid were classified as having a minor relapse.

\section{Statistical analysis}

Disease-related variables including the clinico-serologic subtypes were compared between those who relapsed versus those who did not. Disease duration, activity and damage (physician assessment) status at the time of enrolment, number of relapses, number of prior hospitalizations and major organ involved were also compared between those who consulted and those who did not. We further explored the socio-demographic differences (income, education, employment status, distance from our institute) between them by intergroup comparisons. All values are in median and inter-quartile range. Non-parametric tests were used. SPSS version 26 was used for analysis.

\section{Results}

\section{Demographics}

Of 136 patients (54\% of 250 in MyoCite cohort) with IIM who could be reached, $71(52.2 \%)$ of age 38 years
(F:M=4.5:1), sought 151 median $2(1-3)$ teleconsultations over 93 days (Table 1). Nearly one-third (38\%) consulted on an emergency basis. In contrast, 65 patients (47.8\%) did not respond to our e-mail or messages.

\section{Patient outcomes}

Over a quarter $(26.8 \%)$ of those relapsed over this period; $15.5 \%$ of which were minor and $11.3 \%$ major. The relapses were the highest in JDM (71\%) followed by polymyositis (40\%). Patients who relapsed had more frequent bulbar weakness [7 (39\%) vs 6 (11\%), OR $4.8(1.3-17), p 0.01]$ and heliotrope rash [7 (39\%) vs 7 (14\%), OR 4 (1.2-14), $p$ 0.02] at baseline. There were no differences in socioeconomic factors, MSA and ANA patterns between the two groups. 73.2\% patients were in remission at the time of teleconsultation.

\section{Teleconsultation advice}

In one half (49\%), the same drugs were continued at same doses (Fig. 1b). In the remaining, 11 (15\%) needed increase in drug dose, $12(17 \%)$ needed addition of new drugs and $8(11 \%)$ had drug dose reduction. Various interventions advised are summarized in Table 1.

Those in remission were advised to continue the same treatment $(35,49.3 \%)$ or reduce immunosuppression $(8$, $11.3 \%$.

\section{Patient variables influencing teleconsultations}

Patients of juvenile dermatomyositis (JDM) [71.4\%, OR 8.9 (1.5-51), $p$ 0.014] had increased frequency of relapses compared to other IIM subtypes. The number of consultations did not differ among different clinical subtypes of adults with IIM ( $p=0.144)$ and based on myositis-specific autoantibodies $(p=0.287)$. Patients who relapsed required more consultations $[2(2-3)$ vs $1(1-2), p 0.009$, minor relapses $2(2-3)$ vs $1(1-3), p 0.041]$ over the observation period.

The demographic and socioeconomic profile of the patients who had at least one consultation $(n=71)$ and those who did not $(n=65)$ were compared and no significant differences were observed (Table 2). Teleconsultation-seeking behavior did not differ based on baseline disease-related parameters, such as duration, IIM subtype, major organ involved, prior relapses, distance from home and number of prior hospitalizations.

\section{Logistic issues}

A proportion of consultations sought logistic information alone (15\%), the major issue being non-availability of drug during the pandemic (1.65\%) (Table 1). 
Table 1 Characteristics of patients of IIM who sought teleconsultation

\begin{tabular}{|c|c|}
\hline & $\begin{array}{l}\text { Number } \\
\text { out of } 71 \\
(100 \%)\end{array}$ \\
\hline Total number of consults & 151 \\
\hline \multicolumn{2}{|l|}{ Number of consultations } \\
\hline One & $35(49.3)$ \\
\hline Two & $17(23.9)$ \\
\hline Three & $10(14.1)$ \\
\hline Four & $7(9.9)$ \\
\hline Twelve & $2(2.8)$ \\
\hline Median duration after lockdown (days) & $44(17-74)$ \\
\hline Median interval between consults (days) & $7(7-12)$ \\
\hline \multicolumn{2}{|l|}{ Diagnosis } \\
\hline Dermatomyositis & $22(31)$ \\
\hline Overlap myositis & $19(26.8)$ \\
\hline Anti-synthetase syndrome & $17(23.9)$ \\
\hline Juvenile dermatomyositis & $7(9.9)$ \\
\hline Polymyositis & $5(7)$ \\
\hline Necrotizing myositis & $1(1.4)$ \\
\hline \multicolumn{2}{|l|}{ Disease status } \\
\hline Remission & $52(73.2)$ \\
\hline \multicolumn{2}{|l|}{ Relapse } \\
\hline Minor & $11(15.5)$ \\
\hline Major & $8(11.3)$ \\
\hline \multicolumn{2}{|l|}{ Frequency of relapse among IIM subtypes } \\
\hline Dermatomyositis & $4(18.2)$ \\
\hline Overlap myositis & $3(15.8)$ \\
\hline Anti-synthetase syndrome & $4(23.5)$ \\
\hline Juvenile dermatomyositis & $5(71.4)$ \\
\hline Polymyositis & $2(40)$ \\
\hline Necrotizing myositis & 0 \\
\hline \multicolumn{2}{|l|}{ Type of consultation } \\
\hline \multicolumn{2}{|l|}{ Routine } \\
\hline On time & $48(67.6)$ \\
\hline Delayed & $5(7)$ \\
\hline Emergency & $27(38)$ \\
\hline \multicolumn{2}{|c|}{ Advice (per patient over the observation period) } \\
\hline Continue same treatment & $35(49.3)$ \\
\hline \multicolumn{2}{|l|}{ Intervention } \\
\hline Stop drug & $3(4.2)$ \\
\hline Add drug & $12(16.9)$ \\
\hline Reduce drug dose & $8(11.3)$ \\
\hline Increase drug dose & $11(15.5)$ \\
\hline Others & $2(2.8)$ \\
\hline Infusion & $13(18.3)$ \\
\hline Admission & $5(7)$ \\
\hline Consult local physician & $10(14.1)$ \\
\hline Consult specialist & $3(4.2)$ \\
\hline Review with investigations & $28(39.4)$ \\
\hline
\end{tabular}

Table 1 (continued)

Number out of 71 $(100 \%)$

Logistic issues

$\begin{array}{ll}\text { Drug not available } & 8(11.3) \\ \text { Reimbursement and funding } & 2(2.8)\end{array}$

\section{Challenges encountered}

The challenges encountered include outdated phone numbers, recording outcome measures, language and communication barriers, poor quality and lighting while taking pictures and videos, dealing with the technology gap and accessibility to laboratory services which were dealt with in specific manners as enlisted in Table 3. Certain problems were anticipated but not encountered.

\section{Discussion}

Our prospective evaluation of a cohort of IIM in the early nationwide lockdown period suggests that teleconsultation may be a feasible means of remote evaluation of disabling and severe chronic RDs in a LMIC. Nearly half of our patients sought a teleconsultation in the early pandemic period, of which one-third were for emergencies. A quarter of the IIM patients who reported had experienced a relapse of the disease. Of these, nearly half had a major relapse, mandating high-dose immunosuppression and more frequent remote evaluation. It is noteworthy that children with JDM reported higher relapses compared to adult IIM. Teleconsultation-seeking behaviour was independent of disease duration, IIM subtype, major organ involvement, prior relapses, distance of the hospital from home, and previous hospitalizations.

The early pandemic period was marked by widespread anxiety and concerns of a higher risk of COVID-19 in patients with RDs. There was also a concern of virusinduced relapses in this population, as supported by endogenous and exogenous viral activation in the pathogenesis of several RDs. [1] While such an after-effect has not been demonstrated in other RDs yet, the relapse rate in IIM in the current study is higher compared to previous literature relevant to the disease. $[13,14]$ This could be due to logistic issues in drug procurance during the lockdown, or the inability of patients to renew prescriptions on time. Alternatively, anxiety and stress due to poor preparedness for a black swan event like this could contribute to poor drug compliance, culminating in relapses. [15] Further, a recent study identified enrichment of immunogenic linear epitopes mapping to 20 Coronaviridae species including human SARS-CoV-2 in 
Table 2 Comparison of demographic and socioeconomic profiles of IIM patients who sought a teleconsultation and those who did not

\begin{tabular}{|c|c|c|c|}
\hline & Those who consulted $(n=71)$ & Those who didn't consult $(n=65)$ & $p$ value \\
\hline Gender (M:F) & $1: 4.46$ & $1: 3.33$ & 0.492 \\
\hline Median age (years) & $38(24-46)$ & $36(24.5-46)$ & 0.960 \\
\hline Median disease duration (months) & $30.25(12.34-58.15)$ & $30.3(12.47-55.40)$ & 0.912 \\
\hline Family income (rupees) & $25,000(10,000-56,250)$ & $30,000(14,250-50,000)$ & 0.806 \\
\hline Distance of hometown from hospital (Km) & $173(92-326)$ & $210(93-351)$ & 0.524 \\
\hline \multicolumn{3}{|l|}{ Education $(n=64,57)$} & \multirow[t]{8}{*}{0.531} \\
\hline Primary school & $7(11)$ & $4(7)$ & \\
\hline High school & $6(9)$ & $5(8)$ & \\
\hline Higher secondary & $12(18)$ & $11(19)$ & \\
\hline Graduate & $13(20)$ & $17(29)$ & \\
\hline Post graduate & $23(36)$ & $13(23)$ & \\
\hline Doctorate & $0(0)$ & $1(2)$ & \\
\hline No formal education & $3(5)$ & $6(10)$ & \\
\hline \multicolumn{3}{|l|}{ Employment $(n=60,61)$} & \multirow[t]{7}{*}{0.720} \\
\hline Professional & $9(15)$ & $12(19)$ & \\
\hline Clerical & $5(8)$ & $1(2)$ & \\
\hline Skilled labour & $4(7)$ & $3(5)$ & \\
\hline Student & $16(27)$ & $12(20)$ & \\
\hline Housewife & $31(52)$ & $32(52)$ & \\
\hline None & $1(2)$ & $1(2)$ & \\
\hline \multicolumn{4}{|l|}{ Type of IIM $n(\%)$} \\
\hline $\mathrm{DM}$ & $21(29)$ & $29(44)$ & \multirow[t]{6}{*}{0.289} \\
\hline PM & $5(7)$ & $5(7)$ & \\
\hline ASS & $17(24)$ & $11(17)$ & \\
\hline $\mathrm{OM}$ & $20(28)$ & $11(17)$ & \\
\hline JDM & $7(10)$ & $9(12)$ & \\
\hline IMNM & $1(1)$ & $0(0)$ & \\
\hline \multicolumn{3}{|c|}{ Disease activity at the time of recruitment $n(68.61 \%)$} & \multirow[t]{4}{*}{0.593} \\
\hline Active & $30(44)$ & $21(34)$ & \\
\hline Inactive & $6(9)$ & $7(11)$ & \\
\hline Grumbling & $32(47)$ & $32(52)$ & \\
\hline \multicolumn{3}{|c|}{ Damage assessed by physician at the time of recruitment $n 38.40(\%)$} & 0.47 \\
\hline Minimal & $14(37)$ & $20(50)$ & \multirow[t]{4}{*}{7} \\
\hline Mild & $14(37)$ & $14(35)$ & \\
\hline Moderate & $9(23)$ & $6(15)$ & \\
\hline Severe & $1(3)$ & $0(0)$ & \\
\hline Number of past relapses & $0(0-1)$ & $0(0-1)$ & 0.236 \\
\hline \multicolumn{3}{|l|}{ Major organ involved $n(69.63 \%)$} & \multirow[t]{5}{*}{0.553} \\
\hline Muscle & $44(64)$ & $46(73)$ & \\
\hline Lung & $20(29)$ & $16(25)$ & \\
\hline Heart & $1(1.4)$ & $0(0)$ & \\
\hline Renal & $4(6)$ & $1(1.5)$ & \\
\hline Number of hospitalizations so far & $2(1-4)$ & $1(1-3)$ & 0.091 \\
\hline
\end{tabular}

causing dermatomyositis. Although it is too early to draw firm conclusions from this preliminary data, the possibility of asymptomatic virus infections in patients leading onto relapses of IIM merits further exploration. [16] Even though none of our patients had symptomatic COVID-19, an asymptomatic infection cannot be ruled out. At this juncture, it seems logical that rheumatologists remain aware of the possibility of relapses of IIM after COVID-19.

We observed a higher relapse rate in children as compared with adults. This is surprising given the better outcomes in 


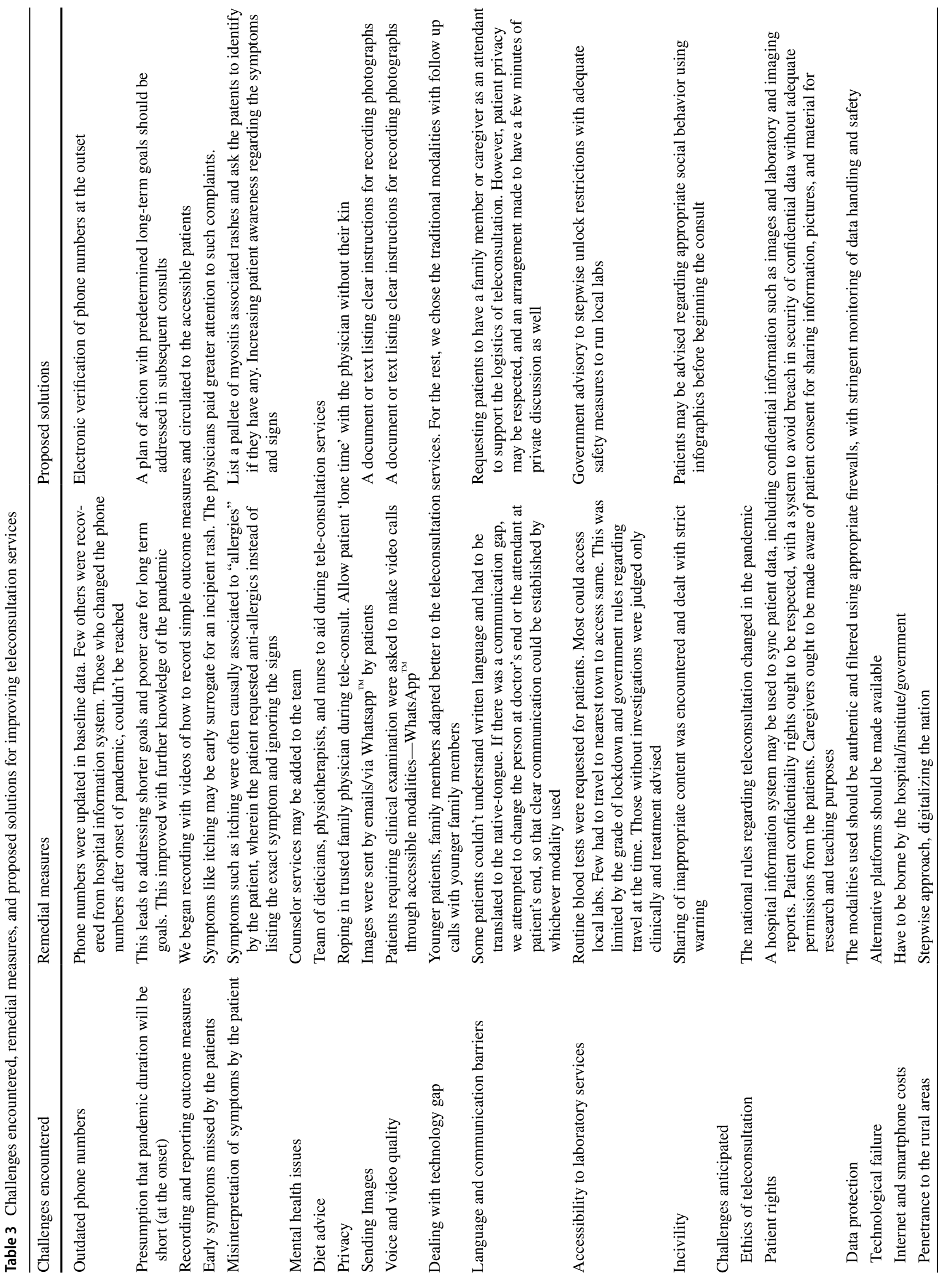


children than adults otherwise. However, jDM is rare, as also reflected by the limited number of patients in the current study. A large proportion of jDM is likely to be managed my paediatricians, and paediatric neurologists. Thus, the study setting at a tertiary-care centre may account for a referral bias of more severe or refractory cases. This can be ascertained by larger collaborative multicentre studies.

While surgical specialities may be limited in their capacity to deliver teleconsultations, several medical specialities may do well on remote consultations. Recently Ahmed et al. concluded that over $70 \%$ of rheumatology consults could be satisfactorily managed remotely after their extensively review across 5 medical specialities. [17] The appropriateness of e-consult inquiries was assessed with 4 parameters: not answerable by reviewing evidence-based summary sources ("point-of-care resource test"), not merely requesting logistic information, having appropriate clinical urgency, and having appropriate patient complexity. These metrics were published at a date beyond our study inception date, are very insightful of such a study design, which we would like to incorporate in subsequent similar studies. The assessment of disease remotely is fraught with challenges due to limited examination and environmental determinants of good photograph and video quality in the event of a relapse. While transitioning from physical to virtual care, it is imperative to design and validate patient-reported-outcome measures specific to the disease. In the study group, the authors later resorted to two simple makeshift-outcome measures-the two-minute walk distance (in metres) and the time to life arms overhead ten times (in seconds) for serial monitoring. Since IIM are heterogenous, a multidisciplinary approach with all stakeholders including patients, physiotherapists, dermatologists, neurologists, pulmonologists, paediatricians, internists and rheumatologists may be involved in developing satisfactory and valid PROs for remote monitoring in the future.

Sizeable number of patients in our study sought advice regarding infusions. Previously disrupted physiotherapy has been a concern among patients with IIM during the pandemic. [18] In the future, telehealth may be improved upon to deliver ancillary care, such as information on day admissions services, physiotherapy, diet and nutrition, and yoga or mindfulness. The future is to guide the patients of established diagnosis (like IIM) to follow up via teleconsultation with recorded outcome measures, nurse-led care (NLC) and tele-triaging. This can be aided by delivery of drugs via postal services for those who cannot procure them from their place. Smart phone apps have evolved during the pandemic to suit the current needs. With metrics like Mobile Application Rating Scale (MARS) [19] which assesses the various mobile apps for overall quality, engagement, functionality, aesthetics, and information, these may be more useful to deliver teleconsultation services across the [20,21]. 
The concept of tele-triaging (triaging in teleconsultation) has to be practiced in e-consults and has been emphasised in other studies too $[3,22]$. NLC has outperformed physiciandriven remote consultation in rheumatoid arthritis in Africa and Middle East countries in a few studies. [23] Further, NLC and teleconsultation services have reduced the proportion of inappropriate calls and overall cost of teleconsultation in a UK based study. [24] While it is too early to speculate if NLC will be useful in managing complex conditions, such as IIM, setting up such services at various tiers of Indian health care system may be in sync with sustainable health sector development goals, and improve care delivery in the prevailing pandemic or those that may arise in future.

It is noteworthy that nearly half of the cohort did not tele-consult in the early pandemic period. The analysis of patients who did not consult, based on their last consultation with us, revealed no differences in teleconsultation seeking behavior (disease duration, IIM subtype, major organ involvement, prior relapses, distance from home and hospitalizations). Not seeking tele-consults, could be because they either they did not have the know-how to use the technology or were complacent due to longstanding remission or too sick to respond to our call. Further, one-third consulted on emergency basis. This emphasizes the need for effective patient education in IIM. Knowing when to ask for help and how to do it, could bring a remarkable difference in patient outcomes. Interestingly, most of the patients hailed from rural places and were on an average $200 \mathrm{~km}$ away from the institute. Further, distance from home was not a determinant of teleconsultation. However, this could be a factor in whether patient wants to continue teleconsultations after the pandemic resolves. The most important challenge in establishing teleconsultation services was the presumption that the pandemic duration is short, and that teleconsultation is a temporary bridge. This led to significant disregard for delivery of wholesome patient care initially. As the wisdom regarding the pandemic increased, the preparedness and care for subsequent consults improved.

Assessing rural and remote access disparities for patients of established rheumatoid arthritis via video-conferencing has revealed no evidence of a difference in effectiveness between inter-professional video-conferencing and traditional rheumatology clinic for both effective follow-up care and patient satisfaction. [8] The main caveat of the study is that sizeable proportions did not seek a consultation. A deeper understanding of patient behaviours, logistic, socioeconomic and cultural determinants can be obtained by interviewing the non-respondents. This may be possible on retrieving the correct phone numbers or interviewing them in person when they visit the clinics post pandemic. A longer follow-up duration is likely to provide greater insight into means to improve remote consultation to tide over the pandemic period.

The ongoing pandemic mandates an acceptance of teleconsultation in the face of adversity. It offers a cost-efficient model for penetration of outreach clinics to the community, in a country with a dismally low physician-patient ratio. Internet coverage has significantly improved in distant villages, and among social media platforms (SMPs), WhatsApp usage is widespread and preferred. [1, 25] A fair acceptance of SMPs for telecare among $90 \%$ rheumatologists has been documented previously. $[3,26]$ Teleconsultation is an evolving field, and no amount of planning is deemed perfect in delivering care in a pandemic. However, our study provides fresh insights into Tele-care in a large and well-characterised cohort of IIM, which are likely to be useful to other centres catering to myositis patients worldwide.

To conclude, virtual consulting can be successfully used in IIM, albeit with a few challenges. Tele-care may be harnessed with the vision to follow tele-triage, advance telesorting to ease rush of cases post-pandemic, formulate community outreach through a structured referral system, and deliver ancillary care. Further, the use of smartphone apps and wearable devices can improve objective recording of simplified patient-reported-outcome measures, while overcoming the constraints of distance and time. Tele-services may be the missing link that interconnect the patient, the primary care physician and the specialist (Figs. 1c, 2).

\section{Conclusion}

Voice-based teleconsultations may be useful to diagnose and manage relapses which are frequent in IIM during the pandemic. Patient education for meticulous and timely reporting may be improve outreach, care, and larger multicentre studies may identify subsets of IIM that require greater care and early tele-triage for effective management of the condition. 


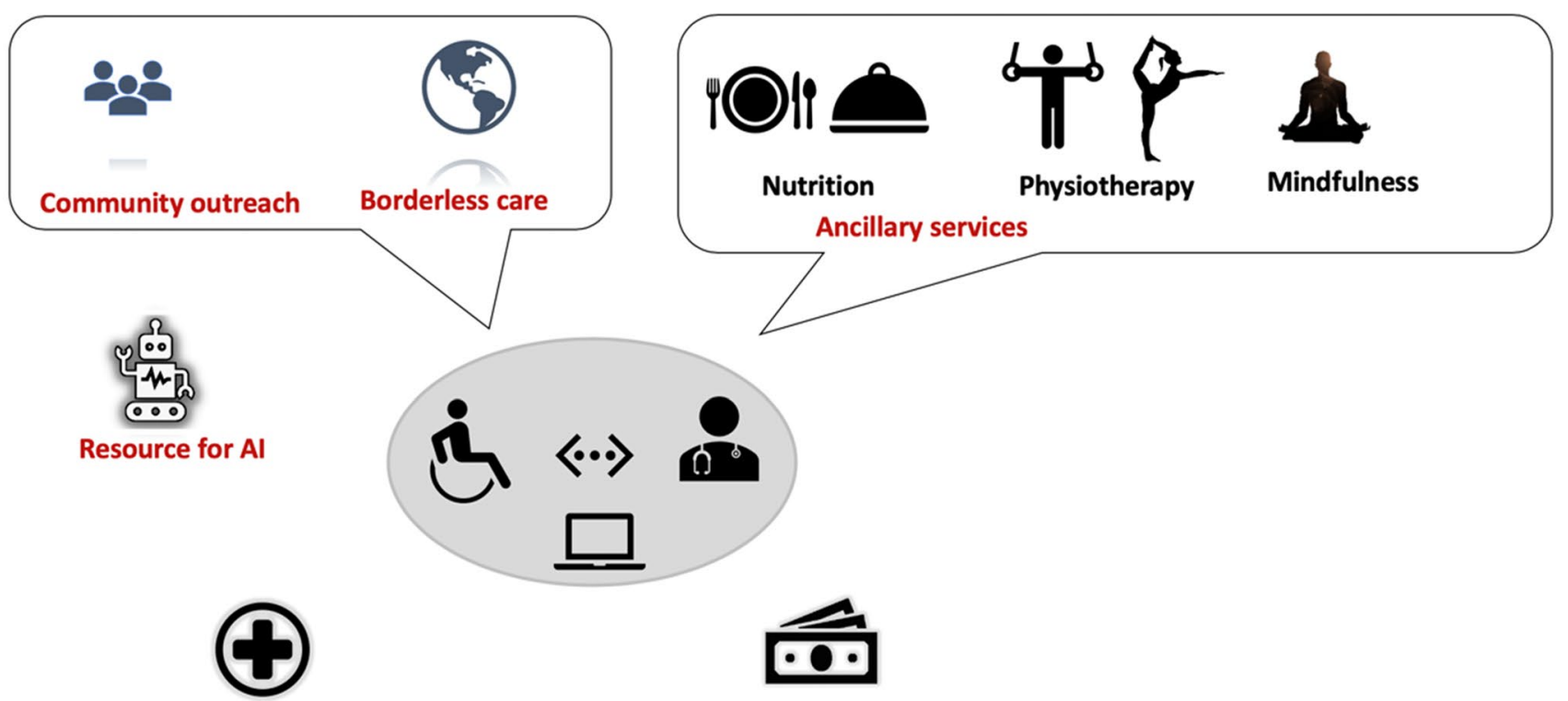

Reduced burden of healthcare

Financially sustainable

Fig. 2 Advantages of the virtual consultation model for the delivery of Healthcare and ancillary services feasible through this approach

Author contributions All authors were involved in ideation, data collection and manuscript preparation. All agree with the submitted version of the manuscript, take responsibility for the content of the entire manuscript, and affirm that any queries related to any aspect of the same are appropriately managed.

Funding This study was not funded.

\section{Compliance with ethical standards}

Conflict of interest The authors declare that there is no conflict of interest relevant to the manuscript.

Ethical approval information Approval obtained from the institute ethics committee of SGPGIMS, Lucknow as per local guidelines (201741-IP-76).

Data sharing statement All data pertaining to the study is included in the manuscript and as supplementary material.

Patient and public involvement The data was partly anonymised and stored as part of an ongoing project (2017-41-IP-76) for which written consent had been obtained previously from the respondents.

\section{References}

1. Gupta L, Misra DP, Agarwal V et al (2020a) Management of rheumatic diseases in the time of covid-19 pandemic: perspectives of rheumatology practitioners from India. Ann Rheum Dis. https ://doi.org/10.1136/annrheumdis-2020-217509
2. Opinc A, Łukasik Z, Makowska J (2020) The attitude of Polish rheumatology patients towards telemedicine in the age of the COVID-19 pandemic. Reumatologia/Rheumatology 58:134-141. https://doi.org/10.5114/reum.2020.96665

3. Gupta L, Misra DP, Agarwal V et al (2020b) Response to: 'telerheumatology in COVID-19 era: a study from a psoriatic arthritis cohort' by Costa et al. Ann Rheum Dis. https://doi.org/10.1136/ annrheumdis-2020-217953

4. Piga M, Cangemi I, Mathieu A, Cauli A (2017) Telemedicine for patients with rheumatic diseases: systematic review and proposal for research agenda. Semin Arthritis Rheum 47:121-128. https:// doi.org/10.1016/j.semarthrit.2017.03.014

5. Masini F, Gjeloshi K, Ferrara R et al (2020) Rheumatic disease management in the Campania region of Italy during the COVID-19 pandemic. RheumatolInt 40:1537-1538. https://doi. org/10.1007/s00296-020-04648-8

6. Mukusheva Z, Assylbekova M, Poddighe D (2020) Management of pediatric rheumatic patients in Kazakhstan during the coronavirus disease 2019 (COVID-19) pandemic. RheumatolInt 40:1351-1352. https://doi.org/10.1007/s00296-020-04613-5

7. Costi S, Caporali R, Cimaz R (2020) Dealing with COVID-19 in a pediatric rheumatology unit in Italy. Paediatr Drugs 22:263-264. https://doi.org/10.1007/s40272-020-00395-2

8. Taylor-Gjevre R, Nair B, Bath B et al (2018) Addressing rural and remote access disparities for patients with inflammatory arthritis through video-conferencing and innovative inter-professional care models. Musculoskeletal Care 16:90-95. https:// doi.org/10.1002/msc. 1215

9. Gupta L, Appani S, Janardana R et al (2019) Meeting report: MyoIN-Pan-India collaborative network for myositis research. Indian J Rheumatol 14:136. https://doi.org/10.4103/injr. injr_40_19

10. STROBE Statement: Available checklists. https://www.strob e-statement.org/index.php?id=available-checklists. Accessed 13 Oct 2020

11. Naveen R, Anuja AK, Rai MK et al (2020) Development of the myocitebiobank: Cost-efficient model of public sector 
investigator-driven biobank for idiopathic inflammatory myositis. Indian J Rheumatol. https://doi.org/10.4103/injr.injr_95_20 ([Epub ahead of print])

12. Mehta P, Gupta L (2020) Combined case record forms for collaborative datasets of patients and controls of idiopathic inflammatory myopathies. Indian J Rheumatol. https://doi. org/10.4103/injr.injr_56_20 ([Ahead of print])

13. Mamyrova G, Rider LG, Ehrlich A et al (2017) Environmental factors associated with disease flare in juvenile and adult dermatomyositis. RheumatolOxfEngl 56:1342-1347. https://doi. org/10.1093/rheumatology/kex 162

14. Wienke J, Bellutti Enders F, Lim J et al (2019) Galectin-9 and CXCL10 as biomarkers for disease activity in juvenile dermatomyositis: a longitudinal cohort study and multicohort validation. Arthritis Rheumatol Hoboken Nj 71:1377-1390. https:// doi.org/10.1002/art.40881

15. Agarwal V, Sharma S, Gupta L et al (2020) (2020) COVID-19 and psychological disaster preparedness - an unmet need. Disaster Med Public Health Prep 25:1-4. https://doi.org/10.1017/ dmp.2020.219

16. Megremis S, Walker TDJ, He X et al (2020) Antibodies against immunogenic epitopes with high sequence identity to SARSCoV-2 in patients with autoimmune dermatomyositis. Ann Rheum Dis. https://doi.org/10.1136/annrheumdis-2020-217522

17. Ahmed S, P. Kelly Y, R. Behera T et al (2020) Utility, appropriateness, and content of electronic consultations across medical subspecialties. Ann Intern Med. https://doi.org/10.7326/M19-3852

18. Gupta L, Lilleker JB, Agarwal V, Chinoy H, Aggarwal R (2020) COVID-19 and myositis- unique challenges for patients. Rheumatology. https://doi.org/10.1093/rheumatology/keaa610 ((Published ahead of print))

19. Davalbhakta S, Advani S, Kumar S et al (2020) A systematic review of smartphone applications available for corona virus disease 2019 (COVID19) and the assessment of their quality using the mobile application rating scale (MARS). J Med Syst 44:164. https://doi.org/10.1007/s10916-020-01633-3

20. Kataria S, Ravindran V (2018) Digital health: a new dimension in rheumatology patient care. RheumatolInt 38:1949-1957. https ://doi.org/10.1007/s00296-018-4037-x

21. Wolthers TO, Wolthers OD (2020) Telephone consultation as a substitute for face-to-face consultation during the COVID-19 pandemic. Dan Med J 67:A04200300 (PMID: 32734880)

22. Rowe-Setz G, Behringer W, Roland D et al (2020) Fifteen minute consultation: when can I use a medical app? Arch Dis Child: EducPract Ed. https://doi.org/10.1136/archdischild-2020-319448 ((edpract-2020-319448))

23. Uthman I, Almoallim H, Buckley CD et al (2020) Nurse-led care for the management of rheumatoid arthritis: a review of the global literature and proposed strategies for implementation in Africa and the Middle East. RheumatolInt. https://doi.org/10.1007/s0029 6-020-04682-6

24. Tomlinson P, Heaton H, Medcalf P et al (2019) A nurse-led rheumatology telephone advice line: service redesign to improve efficiency and patient experience. Br J Nurs 28:619-627. https://doi. org/10.12968/bjon.2019.28.10.619

25. Goel A, Gupta L (2020) Social media in the times of COVID19. JCR J ClinRheumatol 26:220-223. https://doi.org/10.1097/ RHU.0000000000001508

26. Ahmed S, Gupta L (2020) Perception about social media use by rheumatology journals: survey among the attendees of IRACON 2019. Indian J Rheumatol. https://doi.org/10.4103/injr.injr_15_20

Publisher's Note Springer Nature remains neutral with regard to jurisdictional claims in published maps and institutional affiliations. 